gen betrug nicht 9534 , sondern über 9300 Mann, und die Zahl der von Daśui betreuten Bevölkerung 3534 Mann.

Liŭ Maŭ-Tsai

\title{
Michael Bau
}

\section{Politische Partizipation und Entwicklung}

Kritik theoretischer und empirischer Befunde zur Politischen Partizipationsforschung unter besonderer Berücksichtigung der politischen Partizipation marginaler städtischer Gruppen in Lateinamerika

Esprint-Verlag Heidelberg, 1981, Heidelberger Dritte Welt Studien, Band 11, DM 26,-

Die Untersuchung möchte "einen Beitrag leisten zur Diskussion um die Möglichkeiten und Bedingungen einer wirksamen Teilnahme marginaler Gruppen - insbesondere in sogenannten Entwicklungsländern - an der sozio-ökonomischen und politischen Entwicklung ihrer Länder" (S. V.). Um dies zu erreichen, werden Methoden und Prämissen der amerikanischen Partizipationsforschung, soweit sie die lateinamerikanischen "Marginalen" betreffen, kritisch durchleuchtet und ihre Ergebnisse werden im Hinblick auf die Frage untersucht, welche Faktoren auf der Ebene der individuellen Einstellungen, der unmittelbaren Sozialisationserfahrungen und des gesamtgesellschaftlichen Umfelds dazu beitragen, die politische Partizipation der marginalisierten Bevölkerung aus dem Bereich der individuellen Problemlösung herauszuheben und auf die Ebene gesellschaftlicher Veränderungsprozesse zu bringen. Es mag zwar enttäuschen, daß die Literaturauswertung nicht viel mehr bringt als eben diese Fragestellung, die als Forschungsprogramm formuliert wird, doch erweist sich die Aufarbeitung der einschlägigen empirischen Untersuchungen insofern als gerechtfertigt und nützlich, als die am Begriff der Abhängigkeit orientierten Untersuchungen mit ihren Uberwindungsstrategien diese Forschungsergebnisse bislang ignoriert haben.

Dies mag daran liegen, daß die amerikanische, empirische Partizipationsforschung stark einem modernisierungstheoretischen Interesse unterworfen war und politische Partizipation vorwiegend unter dem Aspekt der politischen Integration und Stabilität betrachtete. Zwar ist der Autor einem "kritisch-emanzipativen Demokratiebegriff" verpflichtet und daher gegen diese Art Schlagseite gefeit, doch hindert ihn dies nicht daran, den Nutzen der Partizipationsforschung für auf Veränderungen angelegte Entwicklungsstrategien zu erkennen. Gleichwohl ist anzumerken, daß die - berechtigte - Kritik an den Defiziten und an der Neigung zum ökonomischen Determinismus des überwiegenden Teils der Dependenzforschung zu einem Verzicht auf die Analyse der ökonomischen Randbedingungen von politischer Partizipation überhaupt geführt hat. Daß gerade auch auf der Mikro-Ebene von Stadtteilbewegungen eine gründliche Analyse der ökonomischen Entwicklung nicht nur nützlich, sondern unerläßlich ist, haben u. a. die neueren Arbeiten von $\mathrm{T}$. Evers gezeigt.

Andreas Boeckh 